\title{
A Judgment Criterion of Crossing Obstacle Ability for Lunar Rover with Track Angle

\author{
Kanfeng $\mathrm{Gu}^{\mathrm{a}}$, Guoyong Yang
}

Shenyang Institute of Automation, Chinese Academy of Sciences, Shenyang 110016, China

a kfgu@sia.cn, b gyyang@sia.cn

Keywords: Mobile robot, traversing obstacles, Track angle, Judgment criterion, Torque optimization, Rocker bogie

\begin{abstract}
The problem of traversing obstacles for a six-wheeled rocker bogie lunar rover is presented. Based on the analysis of forces and rover movement track angle, a criterion to determine traversing obstacles ability for the rover is developed to predict whether the robot can overcome current obstacle. The concept of traversing obstacles ability index is presented, which can be used to describe the trafficability of the rover quantificationally. The caculated results of wheels driving torques by this method can be used as a reference for confirming appropriate driving torque with the wheels during they challenge the terrain. A numerical example is given to illustrate our method.
\end{abstract}

\section{Introduction}

Mobile robots are increasingly being employed in rough, outdoor terrain for applications. These applications often require robots to travel across unprepared, rugged terrain to inspect a location or transport material. Some people have studied about the lunar rover on the ability of crossing obstacle. They have got a model of 6 -wheeled rocker bogie lunar rover based on statics and make some reaserch about its ability of climbing vertical obstacles ${ }^{[1,2]}$. But the height of barrier is limited (less then the wheel radius). In another quasi-statics model ${ }^{[3,4]}$, they did not consider the coordination between the wheels and topographical factors. Our paper presents a method to estimate the cantact angle of every wheel based on kinematics. Terrian contact angle is used to describe the movement of the mobile subsystem to find an troque optimization index which contains contact angle and cooperation troque of wheels. Then the judgment criterion of crossing obstacle ability is established to estimate and distinguish the crossing ability quantitatively with obstacle crossing index. At last, a calculation example is presented to illustrate this method.

\section{Dynamic model of lunar rover}

Construction of six-wheeled rocker bogie lunar rover. In this paper, a 6-wheeled rocker bogie lunar rover (Fig. 1) ${ }^{[5]}$ made by Shenyang Institute of Automation of the Chinese Academy of Sciences is studied. The rover has six independently driven wheels mounted on an articulated frame connected to the main body. Each rocker has a rear wheel connected the secondary rocker, called a bogie, connected to the other. The bogie is connected to the rocker with a free pivoting joint. The rockers are connected to the main body with a differential. Fig. 2 presents the parameters of the moving subsystem. $1_{1} \sim 1_{6}, \alpha_{0}, \beta_{0}, \gamma_{0}, \alpha_{1}, \beta_{1}, \gamma_{1}$ are structure parameters of the system. 




Fig. 1. Six-wheeled rocker bogie lunar rover

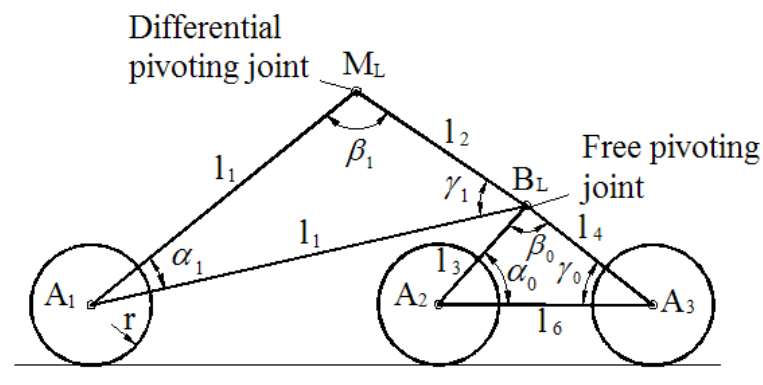

Fig. 2. Parameters of rocker bogie

Dynamic model of lunar rover. We introduce the following assumptions: 1)all theses six wheels is considered as smooth rigid wheel, and they are the same both the mass and the geometric parameters, There is point contact between the wheel and the ground, 2)compared to the main body, the mass of the moving subsystem can be neglected, 3) the velocity of the rover is very small $(\leq 0.05 \mathrm{~m} / \mathrm{s}), 4)$ no steering operarion is imposed on the rover.

The rocker bogie mechanism is modeled as a two dimension system. Here, we consider the planar view of the rover on rough terrain, shown in Fig.3. For the left rocker bogie system:

$\mathrm{P}_{\mathrm{i}}(\mathrm{i}=1,2,3)$ : the contact point of wheel $\mathrm{i}$, respectively

$\delta_{i}(i=1,2,3)$ : the angle of the wheel ground tangent plane respect to the horizon for wheel $i$.

$\mathrm{N}_{\mathrm{i}}(\mathrm{i}=1,2,3)$ : normal force of wheel $\mathrm{i}$

$\mathrm{T}_{\mathrm{i}}(\mathrm{i}=1,2,3)$ : tangential tractive force of wheel $\mathrm{i}$

$\mathrm{F}_{\mathrm{XL}}$ : the force acting on the left differential pivoting joint in the direction of $x$

$\mathrm{F}_{\mathrm{ZL}}$ : the force acting on the left differential pivoting joint in the direction of $y$

Referring to Fig.3, the dynamic force equilibrium

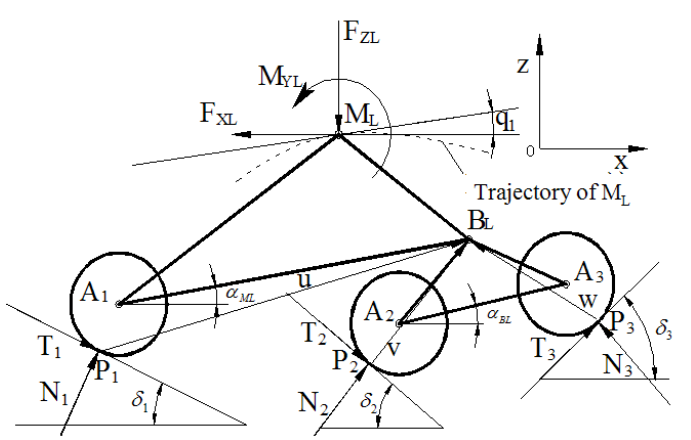

Fig. 3. Force diagram of left rocker bogie system equations in the direction of $\mathrm{X}$ and $\mathrm{Z}$ can be written as:

$$
\sum_{i=1}^{3}\left[\begin{array}{cc}
\cos \delta_{i} & -\sin \delta_{i} \\
\sin \delta_{i} & \cos \delta_{i}
\end{array}\right]\left[\begin{array}{c}
T_{i} \\
N_{i}
\end{array}\right]+\left[\begin{array}{c}
F_{X L} \\
-F_{Z L}
\end{array}\right]=0 .
$$

$\mathrm{B}_{\mathrm{L}}$ is free hinge and can not transmit the torque in the direction of $\mathrm{Y}$. To analyze the rocker and the bogie, we take moments of point $\mathrm{B}_{\mathrm{L}}$ and get the following equilibrium equations:

$$
\begin{aligned}
& \vec{v} \cdot\left[\begin{array}{cc}
\sin \delta_{2} & -\cos \delta_{2} \\
\cos \delta_{2} & \sin \delta_{2}
\end{array}\right]\left[\begin{array}{c}
T_{2} \\
N_{2}
\end{array}\right]+\vec{w} \cdot\left[\begin{array}{cc}
\sin \delta_{3} & -\cos \delta_{3} \\
\cos \delta_{3} & \sin \delta_{3}
\end{array}\right]\left[\begin{array}{c}
T_{3} \\
N_{3}
\end{array}\right]=0 . \\
& \vec{u} \cdot\left[\begin{array}{cc}
\sin \delta_{1} & -\cos \delta_{1} \\
\cos \delta_{1} & \sin \delta_{1}
\end{array}\right]\left[\begin{array}{l}
T_{1} \\
N_{1}
\end{array}\right]+\vec{s} \cdot\left[\begin{array}{l}
F_{X L} \\
F_{Z L}
\end{array}\right]+M_{Y L}=0 .
\end{aligned}
$$

In the equations, the vector from $\mathrm{P}_{1}, \mathrm{P}_{2}, \mathrm{P}_{3}, \mathrm{M}_{\mathrm{L}}$ to $\mathrm{B}_{\mathrm{L}}$ is denoted as $\vec{u}, \vec{v}, \vec{w}, \vec{s}$. The rover moves very slowly, so $\mathrm{F}_{\mathrm{ZL}}$ and $\mathrm{M}_{\mathrm{YL}}$ can be replaced by the gravity and gravity torque shared by point $\mathrm{M}_{\mathrm{L}}$. Then Eq. $1 \sim$ Eq. 3 contain $\mathrm{N}_{1}, \mathrm{~N}_{2}, \mathrm{~N}_{3}, \mathrm{~T}_{1}, \mathrm{~T}_{2}, \mathrm{~T}_{3}$ and $\mathrm{F}_{\mathrm{XL}}$. There are 7 unknown parameters and 4 equations. So we need to find out the optimal value. 
Effective traction coefficient of the ground. Two main index to measure the driving performance are adhesion coefficient $(\varphi)$ and rolling resistance coefficient $(f)$. There are many methods to calculate them on different road with privately models and theories ${ }^{[2]}$. In the equations, there are both the adhesion force $\mathrm{F}_{\mathrm{w}}\left(F_{\mathrm{w}}=N \varphi\right)$ and rolling resistance force $\mathrm{F}_{\mathrm{f}} \quad\left(F_{\mathrm{f}}=N f\right)$. So we may set $\mu=\varphi-f$ and $\mu$ is traction coefficient. Then the effective traction force is :

$$
T=N \varphi-N f=N \mu \text {. }
$$

Wheel-Ground Contact Angle Estimation. To formulate a kinematic model for the rocker bogie system on uneven terrain, a method for estimating the contact angles based on Iagnemma and Dubowsky $^{[6]}$ is implemented in this section.

To the left bogie on uneven terrain, $\alpha_{\mathrm{ML}}$ and $\alpha_{\mathrm{BL}}$ are defined as the angles of main rocker and submain rocker with respectively to the horizon. The wheel center velocities $\mathrm{v}_{\mathrm{A} 1}, \mathrm{v}_{\mathrm{A} 2}$ and $\mathrm{v}_{\mathrm{A} 3}$ are parallel to the wheel-ground tangent plane. $\mathrm{v}_{\mathrm{BL}}$ is the velocity of point $\mathrm{B}_{\mathrm{L}}$. Main rocker is as long as 15. The distance between the centers of wheel 2 and 3 is $1_{6}$, as in Fig. 4.

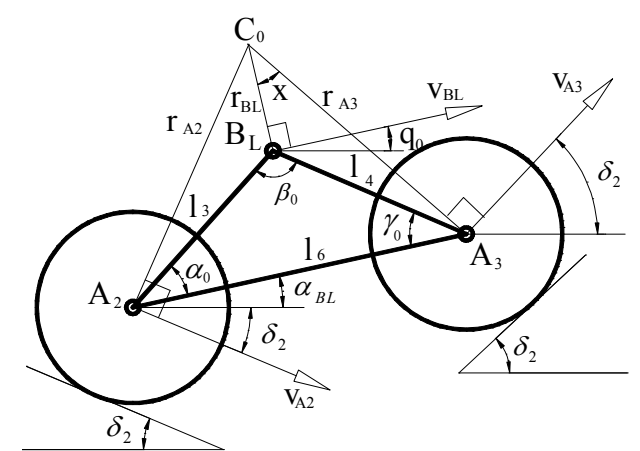

Fig. 4. Kinematices analysis of left bogie based on wheel-ground contact angle

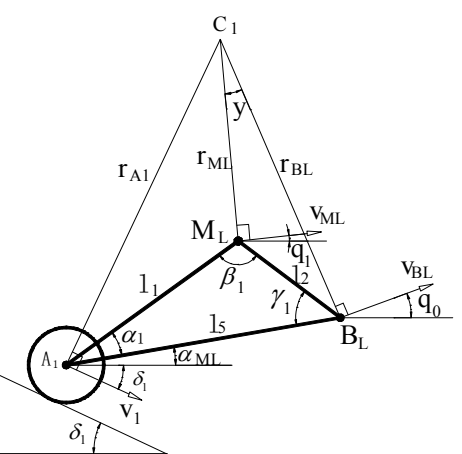

Fig. 5. Kinematices analysis of left rocker based on wheel-ground contact angle

Consider the velocity relation of two points on one rigid body, we get the following equations:

$$
\sin \left[\left(\delta_{2}-\alpha_{B L}\right)-\left(\delta_{3}-\alpha_{B L}\right)\right]=\left(l_{6} \dot{\alpha}_{B L} / v_{A 3}\right) \cos \left(\delta_{2}-\alpha_{B L}\right)
$$

set $a_{1}=l_{6} \dot{\alpha}_{B L} / v_{A 3}, b_{1}=v_{A 2} / v_{A 3}$ then

$$
\begin{aligned}
& \delta_{2}=\alpha_{B L}+\sin ^{-1}\left(\frac{a_{1}^{2}+b_{1}^{2}-1}{2 a_{1} b_{1}}\right) . \\
& \delta_{3}=\alpha_{B L}+\sin ^{-1}\left(\frac{-a_{1}^{2}+b_{1}^{2}-1}{2 a_{1}}\right) .
\end{aligned}
$$

Set $\mathrm{C}_{0}$ and $\mathrm{C}_{1}$ as the instantaneous center of velocities of bogie and main rocker respectively. $\mathrm{r}_{\mathrm{A} 3}, \mathrm{r}_{\mathrm{A} 2}, \mathrm{r}_{\mathrm{A} 1}, \mathrm{r}_{\mathrm{BL}}$ and $\mathrm{r}_{\mathrm{ML}}$ represents the radius of gyration of relative points. The velocity of $\mathrm{B}_{\mathrm{L}}$ is

$$
v_{B L}=\dot{\alpha}_{B L} \sqrt{r_{A 2}^{2}+l_{3}^{2}-2 r_{A 2} l_{3} \cos \left(\pi / 2+\delta_{2}-\alpha_{B L}-\alpha_{0}\right)} .
$$

and $r_{A 2}=l_{6} \sin \left(\frac{\pi}{2}-\delta_{1}+\alpha_{B L}\right) / \sin \left(\delta_{3}+\delta_{2}\right)$

In Fig.5, consider main rocker, we can get similar equations as Eq.5 Eq.7. 
In those formulas, $\alpha_{\mathrm{BL}}, \alpha_{\mathrm{ML}}$ and $\dot{\alpha}_{B L}, \dot{\alpha}_{M L}$ are gotten by gyroscope and inclinometer while $\mathrm{v}_{\mathrm{A} 1}$, $\mathrm{v}_{\mathrm{A} 2}, \mathrm{v}_{\mathrm{A} 3}$ are gotten by encoder. We can get the value of $\mathrm{v}_{\mathrm{BL}}$ by some kinematics calculation.

The calculation of direction angle of trajectory of differential joint center $M_{L}$. The direction angle of trajectory of $\mathrm{M}_{\mathrm{L}}$ is the angle between the trajectory and horizontal line in the direction of movement. The direction of trajectory of point $\mathrm{B}_{\mathrm{L}}$ is

$$
q_{0}=\delta_{3}-\cot ^{-1}\left[\frac{l_{6} \cos \left(\delta_{2}+\alpha_{B L}\right)}{l_{4} \cos \left(\alpha_{B L}-\delta_{3}-\gamma_{0}\right) \sin \left(\delta_{2}+\delta_{3}\right)+\tan \left(\alpha_{B L}-\delta_{3}-\gamma_{0}\right)}\right] .
$$

The direction angle of trajectory of $\mathrm{M}_{\mathrm{L}}$

$$
q_{1}=q_{0}-\cot ^{-1}\left[\frac{l_{5} \cos \left(\delta_{1}+\alpha_{M L}\right)}{l_{2} \cos \left(\alpha_{M L}-q_{0}-\gamma_{1}\right) \sin \left(\delta_{1}+q_{0}\right)+\tan \left(\alpha_{M L}-q_{0}-\gamma_{1}\right)}\right] .
$$

\section{The optimization of driving force and the judgment criterion of crossing obstacle ability}

In kinematics, vehicle is driven by the force between the driving wheel and ground to achieve the expected movement. Coordination and optimization are needed to get the maximum crossing ability since all wheels are initiative driving wheels.

Optimization index of maximum driving force. The optimization index is defined as:

$$
f_{t}=\max \left\{\sum_{i=1,2,3}\left[T_{i} \cos \left(\delta_{i}-q_{1}\right)-N_{i} \sin \left(\delta_{i}-q_{1}\right) \operatorname{sgn}\left(\frac{\pi}{2}-\delta_{i}\right)\right]\right\} .
$$

In the formula, sgn (-) is a signal function, $f_{t}$ is the maximum force of each wheel in the direction of $\mathrm{M}_{\mathrm{L}}$ 's trajectory.

Constraints of optimization. The following constraints is met in Eq.1 Eq.3:

a) positive pressure is non-negative: $\mathrm{N}_{\mathrm{i}} \geq 0,(\mathrm{i}=1,2,3)$;

b) the constraint of driving torque of wheel motor: $\tau_{\mathrm{i}}^{\min } \leq \mathrm{T}_{\mathrm{i}} \mathrm{r} \leq \tau_{\mathrm{i}}^{\max },(\mathrm{i}=1,2,3)$;

c) constraint of thrust force offered by the ground: $\mathrm{T}_{\mathrm{i}}{ }^{\min } \leq \mathrm{T}_{\mathrm{i}} \leq \mathrm{T}_{\mathrm{i}}^{\max },(\mathrm{i}=1,2,3)$

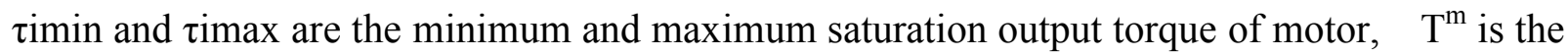
maximum shear stress ${ }^{[8]}$ of soil calculated by Mohr-Coulomb theory. $T^{m}=A c+N \tan \phi$, c and $\phi$ are cohesion and internal friction angle separately, while $\mathrm{N}$ is positive pressure and $\mathrm{A}$ is the wheel-soil contact area.

Judgment criterion of crossing obstacle ability. Define $F_{S}=f_{t}-\left(F_{Z L} \sin q_{1}+F_{X L} \cos q_{1}\right)$. We can judge the crossing ability by the sign of $F_{S}$ : if $F_{S}>0$, the lunar rover can stride the obstacle by distributing driving torque reasonably. The pattern of the judgement is as fallow:

$$
\begin{aligned}
& \text { if } N_{i} \geq 0(i=1,2,3) \text {, and }\left\{\begin{array}{l}
F s>0, Y e s, \\
F s<0, \text { No. . }
\end{array}\right. \\
& \text { if } N_{i}<0(i=1,2,3), \text { No. }
\end{aligned}
$$

Define $F_{S}$ as index of crossing obstacle ability. The bigger $F_{S}$, the stronger ability of crossing obstacle. So $F_{S}$ can estimate crossing ability quantitatively. 


\section{Example}

In fact, the tilt angle of obstacles is less then $90^{\circ}$ generaly. Suppose an obstacle is $0.3 \mathrm{~m}$ in height and the tilt is $\theta=60^{\circ}$. The rover may go throuth the phases (a),(b) and (c) in figure 6 . Here are parameters: $\alpha_{0}=30^{\circ}, \beta_{0}=120^{\circ}, \alpha_{1}=12.8^{\circ}, \beta_{1}=139.6^{\circ}, 1_{1}=1.092 \mathrm{~m}, 1_{2}=0.522 \mathrm{~m}, 1_{3}=0.577 \mathrm{~m}, 1_{4}=0.577 \mathrm{~m}$, $1_{5}=1.528 \mathrm{~m}, 1_{6}=1 \mathrm{~m}, \mathrm{G}=100 \mathrm{~kg}, \mathrm{r}=0.13 \mathrm{~m}, 1_{\mathrm{d}}=0.2 \mathrm{~m}$. Parameters of the soil are: $\mathrm{c}=1.32 \mathrm{KPa}, \varphi=31.4^{\circ} . \mathrm{In}$ 3 different traction coefficients, the curves of $F_{S}$ with different $h x$ in 3 phases are given in Fig.7, Fig. 8 and 9. Define $F_{S}=0$ as the reference curve to make the conclusion. If $F_{S}$ is upper then reference curve, the rover can go throuth the obstacle, and vice versa.

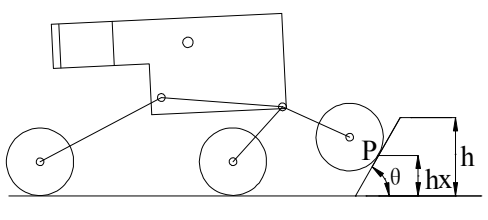

(a) phase 1: wheel 3 is crossing

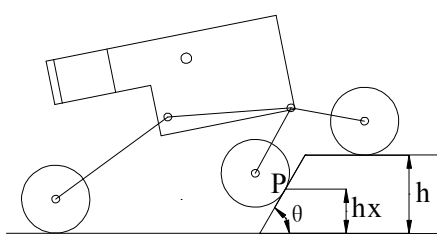

(b) phase 2: wheel 2 is crossing

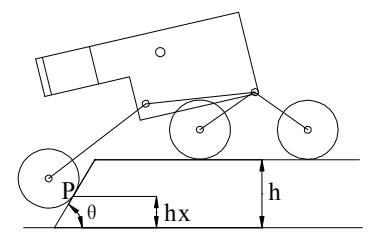

(c) phase 3: wheel 1 is crossing

Fig. 6. Lunar rover is crossing a $300 \mathrm{~mm}$ inheight and $60^{\circ}$ grape

In phase $1(2,3)$, it is appeared in Fig. $7(8,9)$ that when $\mu=0.3$ and $h x<0.1 \mathrm{~m}(\mathrm{hx}<0.12 \mathrm{~m}$ in phase $2,0.27 \mathrm{~m}<\mathrm{hx}<0.3 \mathrm{~m}$ in phase 3 ), $\mathrm{F}_{\mathrm{S}}$ is lower then the reference curve, so the rover can't got across. When $\mu=0.325$, the rover got across the obstacle. If $\mu$ increases to $0.35, \mathrm{~F}_{\mathrm{S}}$ increases upper then reference curve to cross the obstacle. What's more, $\mathrm{F}_{\mathrm{S}}$ will increase with $\mathrm{hx}$. When $\mu$ is certain, $h x=0.065 \mathrm{~m}(\mathrm{hx}=0.065 \mathrm{~m}$ in phase $2, \mathrm{hx}=0.3 \mathrm{~m}$ in phase 3$)$, wheel 3 (wheel 2,1$)$ barely contact the grape, $F_{S}$ get it's minimum. It is the hardest state to cross.

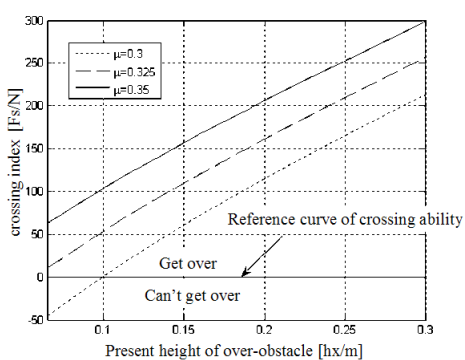

Fig. 7. The curve of crossing terrain index of wheel 3

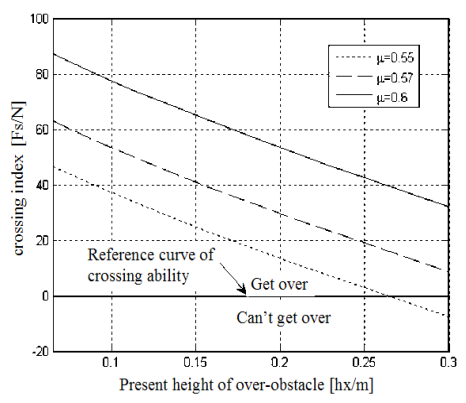

Fig. 9. The curve of crossing terrain index of wheel 1

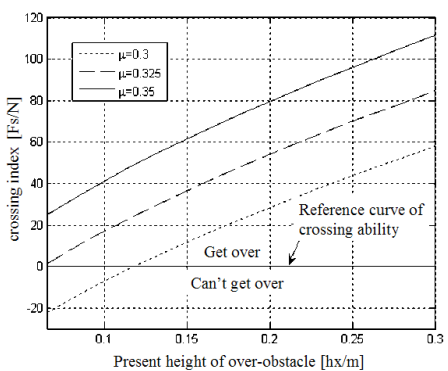

Fig. 8. The curve of crossing terrain index of wheel 2

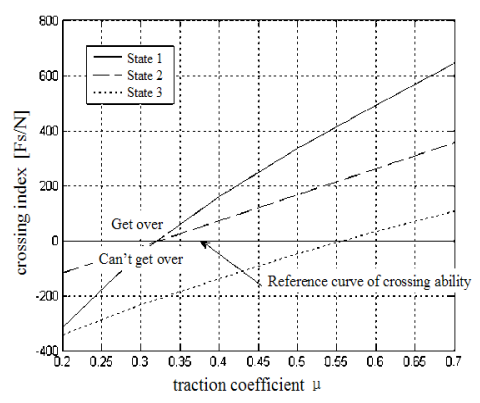

Fig. 10. The curve of crossing index and the traction coefficient of 3 states 
The curve of crossing index and the maximum traction coefficient allowed of 3 states are drawn in Fig.10. So the minimum index is in state 3. The crossing ability of wheel 1 is the minimum which determins the crossing ability of the whole rover. After all, only when the traction coefficient $\mu>0.55$, the lunar rover will get across the grape.

\section{Conclusion}

One of the most important problems is estimating whether it can get across the obstacle when the rover is driving. In this paper, a judgment criterion of crossing obstacle ability for lunar rover is found based on dynamics model and kinematics analysis of wheel-ground contact angle. We also define the crossing index to estimate the crossing ability quantitatively. The result of this paper can guide path planning and control parameters optimization of lunar rover to improve crossing ability.

\section{References}

[1] HU Ming; DENG Zong-quan; GAO Hai-bo; WANG Shao-chun. Analysis of Climbing Obstacle Trafficability on the Six-Wheeled Rocker-Bogie Lunar Rover [J]. Journal of Shanghai Jiaotong University,2005,39(6):928 932.

[2] LI Hai-bin; DUAN Zhi-xin; Kang Bu-xiao. Analysis of Obstacle Capability of Lunar Rover with the Rocker-bogie Structure [J]. Journal of Chongqing Institute of Technology,2005,19(11):1 5.

[3] FARRITOR S, H. HACOT, Dubowsky S. Physics-based planning for planetary exploration. Robotics and Automation[C]. Proceedings. 1998 IEEE International Conference on Robotic and Automation. Cambridge, USA, 1998: 278 283.

[4] IAGNEMMA. K. , RZEPNIEWSKI, A., et al.., Control of robotic vehicles with actively articulated suspensions in rough terrain[J], Autonomous Robots, Vol. 14, No. 1, January, 2003: 5-16.

[5] P. FIORINI, Ground mobility systems for planetary exploration[C], Proceedings of the 2000 IEEE International Conference on Robotics \& Automation, San Francisco, April, 2000: 908-913.

[6] IAGNEMMA. K., S. DUBOWSKY, Traction control of wheeled robotic vehicles with application to planetary rovers, The International Journal of Robotics Research [J] , 2004, 10: 1029 1040.

[7] Ju Hehua Cui Pingyuan. MODELLING AND CONTROL FOR LUNAR ROCKER-BOGIED ROVER WITH SLIP [J], 2005, 41(9):134 139.

[8] J. Y. WONG, Theory of ground vehicles (Third Edition) [M].John Wiley \& Sons,2001: 100 $\sim 130$.

[9] NATIONAL AERONAUTICS AND SPACE ADMINISTRATION, Nasa space vehicle design criteria (Environment), Lunar Surface Models [D], Nasa , 1969: 27-30. 